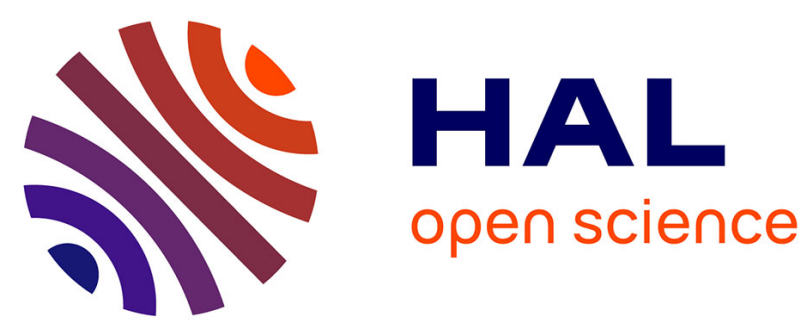

\title{
Novel image analysis method for in situ monitoring the particle size distribution of batch crystallization processes
}

Benoît Presles, Johan Debayle, Gilles Fevotte, Jean-Charles Pinoli

\section{- To cite this version:}

Benoît Presles, Johan Debayle, Gilles Fevotte, Jean-Charles Pinoli. Novel image analysis method for in situ monitoring the particle size distribution of batch crystallization processes. Journal of Electronic Vision, 2010, 19, pp.031207. 10.1117/1.3462800 . hal-00550948

\section{HAL Id: hal-00550948 \\ https://hal.science/hal-00550948}

Submitted on 17 Jan 2011

HAL is a multi-disciplinary open access archive for the deposit and dissemination of scientific research documents, whether they are published or not. The documents may come from teaching and research institutions in France or abroad, or from public or private research centers.
L'archive ouverte pluridisciplinaire HAL, est destinée au dépôt et à la diffusion de documents scientifiques de niveau recherche, publiés ou non, émanant des établissements d'enseignement et de recherche français ou étrangers, des laboratoires publics ou privés. 


\title{
Novel image analysis method for in situ monitoring the particle size distribution of batch crystallization processes
}

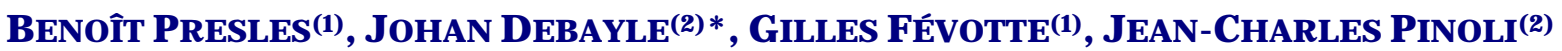

(1) Ecole Nationale Supérieure des Mines de Saint Etienne ; Centre SPIN ; Département GENERIC ; LPMG-UMR CNRS 5148 ; 158 Cours Fauriel, 42023 Saint-Étienne Cedex 2, France

(2) Ecole Nationale Supérieure des Mines de Saint Etienne ; Centre CIS ; Département DIM ; LPMG -UMR CNRS 5148 ; 158 Cours Fauriel, 42023 Saint-Étienne Cedex 2, France

\begin{abstract}
This article presents a new in situ method to monitor the particle size distribution (PSD) during batch solution crystallization processes. Using a new in situ imaging probe, the "EZProbe sensor," real time acquisition of 2-D images of particles during the batch process is now possible. To analyze these images, a novel image analysis method is carried out. First, segmentation and restoration algorithms are performed to identify the particles and thereafter geometrical particle measurements are achieved to obtained the PSD of the batch crystallization process over time. Satisfactory measurements are obtained provided that the overall solid concentration does not exceed a threshold above which too many overlapping crystals make discrimination between particles impossible.
\end{abstract}

Keywords:

particle size distribution ; (PSD) ; image analysis ; 2-D images of particles ; batch crystallization process ; EZProbe sensor ; segmentation algorithms ; restoration algorithms

\section{Introduction}

Solution crystallization processes are widely used in the process industry and notably in the pharmaceutical industry as separation and purification operations and are expected to produce solids with desirable properties. In particular, in terms of pharmaceutical solid ingredients, the size and the shape of crystals are known to have a considerable impact on the final quality of drugs (i.e., bioavailability, stability during storage, ease of processing, etc.). To measure the particle size distribution (PSD); it is well established that conventional monitoring techniques, such as laser diffraction (LD), ultrasonic attenuation spectroscopy (UAS), or focused-beam reflectance measurement (FBRM) do not provide reliable in-line estimates. Major difficulties arise from the use of LD techniques since it requires highly diluted samples of rather ideally shaped particles. Indeed, "ideally shaped" means here that the particles, to fit the theoretical models used to process LD measurements, should be as close as possible to spheres and exhibit rather simple distributions [1] (i.e., multimodal distributions should be very cautiously analyzed). The main disadvantage of UAS is that it requires a large set of accurate physical data related to the liquid and particle phases, [2] which is almost prohibitive for routine industrial applications. Finally, the main disadvantage

\footnotetext{
${ }^{*}$ Corresponding author: debayle@emse.fr
} 
of FBRM is that it does not actually measures the PSD but the chord length distribution (CLD).

One must therefore convert the measured CLD into its corresponding PSD, which is an illposed problem, even though successful experimental applications have been reported for spheres [3] and octahedrons [4]. Various 2-D image based methods also exist, [5-7] but most of them require a priori knowledge and/or assumptions about the shape of the particles. Therefore, the latter methods cannot easily handle crystallization phenomena such as aggregation or breakage of particles. Moreover, those methods do not take into account particles outside the focal plane. Contrary to those methods, this study does not make any assumption about the shape of the particles. Therefore, it can handle particles of any shape, and moreover, it restores out-of-focus particles to maximize the data to be analyzed and to acquire an accurate particle characterization. In the first section, the experimental system used to monitor the crystallization process is explained. In the second section, the image analysis algorithm is developed (image segmentation, restoration and measurement). Finally, typical characterization results are presented before concluding.

\section{Materials, Experimental Setup, and Image Acquisition}

\section{II.1. Materials and Experimental Setup}

Crystallization experiments were performed with citric acid particles crystallizing in water in a 3-L lab-scale batchjacketed crystallizer, equipped with a profiled pale propeller (Mixel TT) and four baffles. The stirring rate was set to $250 \mathrm{rpm}$. The temperature of the crystallizing suspension was controlled by means of hydroalcoholic fluid circulating in the jacket. Isothermal desupersaturation crystallization experiments were started though seeding: sieved citric acid particles were introduced in the crystallizer maintained under supersaturated conditions. To monitor the experiments a new in situ imaging probe, the "EZProbe sensor" [Figure 1(a)], developed at the University of Lyon (Lyon 1) was used. A CCD camera connected to an acquisition interface records transmitted light images. The interface retrieves the video data, compresses it if necessary, and sends it to a computer [Figure 1(b)]. The probe was immersed into the reactor, as shown in [Figure 1(b)] and filmed a restricted volume within the suspension. The system acquires video sequences of 2-D projections of 3-D particles with the following specifications: 25 frames/ s, each frame is $640 \mathrm{x} 480$ pixels 8-bit grayscale bit depth representing $1.3 \times 1 \mathrm{~mm}$. In addition to the in situ camera, the process was monitored using in situ temperature and supersaturation measurements; the latter were performed using attenuated total reflection (ATR) Fourier transform infrared (FTIR) spectroscopy [8-9].

\section{II.2. Image Acquisition}

The new imaging probe enables real-time acquisition of 2-D images of the particles generated during crystallization processes. Figure 2 shows some typical images of citric acid particles during crystallization at different times. To quantitatively characterize the particles, some difficulties must be considered in the different image processing steps: particle clustering, shape heterogeneity, anisotropy, particles outside the focal plane, etc. However, because the image analysis method was designed without any assumption about the shape of the particles and it restores blurred particles, it can handle these phenomena. The next section explains the image analysis method, which was designed so as to deal with these difficulties.

\section{Image Analysis Method: PSD Imaging}

\section{III.1. Image Segmentation}

First, for each image of the video sequence [Figure 3(a)], all particle regions were isolated, as displayed in [Figure 3(b)]. The method here consists of applying the watershed algorithm [10] constrained by the h-minima [10] of the image. Then, the spatial support of each particle was detected [Figure 3(c)] by applying on each delimited area the automatic thresholding 
proposed by Gonzalez and Woods [12]. Second, the particles in the focal plane (in-focus particles) were discriminated from those outside the focal plane (blurred-particles) using a focus measurement [13-15] calculated locally: the variance focus measurement [16] defined as

$$
\frac{1}{M N} \sum_{x=1}^{N} \sum_{y=1}^{M}[I(x, y)-\mu]^{2}
$$

where $I: \Omega \subset \mathbb{Z}^{2} \rightarrow \mathbb{Z}$ is the gray-level image $(x, y \in \Omega), \mathrm{M} \times \mathrm{N}$ is the size of the neighborhood, and $\mu=\left(\frac{1}{M N}\right) \sum_{x=1}^{N} \sum_{y=1}^{M} I(x, y)$ is the mean gray level in the neighborhood. Then, performing a reconstruction process between the "watershed image" and the "variance image", out-of-focus regions and in-focus regions were determined, respectively [Figure 3(d)]. Therefore, at the end of the segmentation two elements of information were obtained: the spatial support and the degree of focus of each particle/ region [17].

\section{III.2. Image Restoration}

To maximize the data to be analyzed and to achieve an accurate particle characterization, a restoration step was performed: particles outside the focal plane were restored. To do so, a blind deconvolution technique was applied to the detected blurred particles/clustered particles. Due to the presence of "ringing effects" in classical blind deconvolution algorithms, [18-20] a new blind deconvolution algorithm based on the image characteristics was developed [21].

About the latter, it is assumed that a particle 1-D profile can be modeled by a perfect square function [Figure 4]. However in practice, it is assumed that only the edge of an in-focus particle is perfect. If holes occur inside the particle image, they are filled. Moreover, it is hypothesized that on each region:

* The particle image is noise free and the blur is spatially invariant; that is, $g(x)=f(x) h(x)$, where $g$ is the gray-level image of the blurred particle resulting from a convolution of $f$, the gray level image of the original particle, and $h$, the point spread function (PSF).

* The blur is weak in the sense that $\max (g)=\max (f)$.

* The PSF $h$ is isotropic and normalized so that $\int_{-\infty}^{\infty} h(x) d x=1$.

Under these hypotheses and using Fubini's theorem, it can be demonstrated that $\int_{-\infty}^{\infty} g(x) d x=\int_{-\infty}^{\infty} f(x) d x$. Consequently, the gray level image $\mathrm{f}$ of the original particle can be approximated by optimization:

$$
f=\underset{p}{\arg \min }\left|\int_{-\infty}^{\infty} p(x) d x-\int_{-\infty}^{\infty} g(x) d x\right|,
$$

where $p$ belongs to the set of the square functions of "height" max $(g)$. To compute the solution of Equation (2), f was calculated by iteratively eroding in the sense of mathematical morphology [22] the spatial support of $g\left(p_{0}, p_{1}\right.$, etc.) [Figure 5]. The blind deconvolution algorithm already described was applied to the already detected blurred particles. The focused particles were not processed. Figure 6 shows the results for a few selected particles from the original image [Figure 3(a)]. 


\section{III.3. Restoration Validation}

To validate the proposed deconvolution algorithm, an image of an isotropic object [Figure 7(a)] and an image of an anisotropic one [Figure 7(b)] were blurred artificially by a normalized Gaussian PSF with a standard deviation $\sigma \in \llbracket 1,5 \rrbracket$. Then, the proposed segmentation step was applied on these images with or without the deconvolution step, and in each case, the normalized root mean square error (NRMSE) was calculated according to $\sigma$ [Figure 7(c)]. As it can be noticed in [Figure 7(c)], whatever the object is and whatever $\sigma$ is, the value of the NRMSE is less important when the algorithm is applied with the restoration step than without it.

\section{III.4. Image Measurement}

As the spatial support of each particle is now well defined (blurred particles in particular), an accurate PSD analysis is possible. To do so, each particle projected area was measured and for each projected area, the diameter of the sphere with the same projected area was calculated. Then, the PSD of the particles was constructed by calculating the normalized histogram of the diameter values. Hence, the particle size is equal to the diameter of the sphere with an equivalent projected area.

\section{Validation and Results}

\section{IV.1. Validation through Computer Simulation}

To validate the PSD imaging method explained in the previous section, an experiment was performed on simulated images. A thousand isotropic particles with random size diameters D ( 2 pixels $\leq \mathrm{D} \leq 60$ pixels) were computationally generated [hence, their PSD was known $\left(\mathrm{PSD}_{1)}\right.$ ]. Then, each particle was blurred artificially by a normalized gaussian PSF with a random standard deviation $\sigma \in \llbracket 1,5 \rrbracket$. Applying the PSD imaging method already explained, the PSD of the particles was recovered $\left(\mathrm{PSD}_{2}\right)$. To achieve reliable statistical data, this process was repeated 100 times and each time, to quantify the error between the two PSDs, the normalized $\mathrm{L}_{1}$ error ( $\mathrm{NL}$ Err) between the two cumulative size distributions $\left(\mathrm{CSD}_{1}\right.$ and $\left.\mathrm{CSD}_{2}\right)$ was calculated $\left\{N L_{1} E r r=100\left[\int\left|C S D_{1}-C S D_{2}\right|\right] / \int C S D_{1}\right\}$. Figure 8 shows an example of CSDs obtained with this simulation. As one can notice, $\mathrm{CSD}_{2}$ fits better $\mathrm{CSD}_{1}$ for particles with diameters larger than 12 pixels. Indeed, for small particles, the hypothesis $\max (g)=\max (f)$ has a greater probability to be unverified. However, on average, the normalized $\mathrm{L}_{1}$ error was only about $2.49 \%$ with a standard deviation of $0.18 \%$.

\section{IV.2. Experimental Validation}

To validate the PSD imaging method explained in the previous section, an experiment was performed using polydisperse silica beads. The PSD of the silica beads was obtained using two different methods: the PSD imaging method presented here and Coulter counter measurements. The Coulter counter is a rather reliable offline device that is currently used for particle sizing and counting, e.g., in the field of biology [23] (blood cells, microscopic algae, etc.). It detects the change in electrical conductance of a small aperture as a fluid containing particles/cells is drawn through. The particle size measured with the Coulter counter is the diameter of the sphere whose volume is equal to that of the particle. The measured particles in this experiment are spherical beads, therefore the diameters measured with the PSD imaging method are comparable to the diameters measured with the Coulter counter. The PSD obtained with the Coulter counter is expressed in terms of volume percentage and for convenience, the bins size was chosen as $4 \mu \mathrm{m}$. By calculating for each diameter obtained with the PSD imaging method the volume of the corresponding bead, it is also possible to express the PSD of the silica beads in terms of volume percentage. Therefore, the PSDs obtained with these two techniques are comparable. The image processing PSD was calculated on 1000 
particles. First, one can notice in Figure 9 that the two PSDs look very similar and more particularly that the main modes obtained with the two different methods are both located around $90 \mu \mathrm{m}$. Moreover, the value of the normalized $\mathrm{L}_{1}$ error between the two cumulative size distributions is only about 3\%.

\section{IV.3. Results}

Figure 10(b) shows a typical result of PSD (PSD is expressed in terms of percentage in number of particles) obtained after processing the raw image displayed in Figure 10(a) using the PSD imaging method explained in the previous section. Both the PSD in the reactor and in the measurement cell are assumed to be homogeneously distributed due to stirring, which maintains the particles in suspension. Five minutes after seeding (i.e., after crystallization the begun), it can be observed that the majority of the particles still exhibit a diameter (the measured diameter corresponds to the diameter of the sphere with an equivalent projected area) smaller than $100 \mu \mathrm{m}$ [Figure 10(b)]. However, note that a peak is present around 167 $\mu \mathrm{m}$, which corresponds to the aggregated particle present in the bottom center of the image [Figure 10(a)]. Since only few particles can be observed in each image, an improvement is obtained after averaging the PSD. It is usually considered that correct particle sampling requires at least 1000 particles to be measured. Therefore, to link the averaging of the PSD to the crystallization process, a particle counter was implemented in the PSD imaging method. Once the particle counter is above the threshold of 1000 particles, an average PSD is computed.

Figure 11(a) (PSD $)$ shows the average PSD of citric acid particles computed after about 2 min of crystallization and Figure 11(b) ( $\mathrm{PSD}_{2}$ ) shows the average PSD of citric acid particles computed after about 5 min of crystallization. As one can notice, due to the development of both crystal nucleation and growth, the PSD spreads and the growth of the initial seed

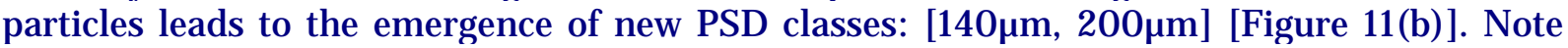
that the average PSD computed after about $5 \mathrm{~min}$ of crystallization is quite different from the PSD calculated at $t=5$ min $23 \mathrm{~s}$ [Figure 10(b)]. This is clearly because the set of particles processed to yield the "instantaneous PSD" at $t=5 \mathrm{~min} 23 \mathrm{~s}$ is too small to achieve an accurate measurement.

In addition to the size distribution, note that shape parameters (elongation, convexity, etc.) could be calculated for each particle, providing information about possible anisotropy of the particles and aggregation processes.

\section{Conclusions}

During this work, a novel image processing method, called PSD imaging, enabling us to estimate the time-varying PSD of particles in suspension was developed and evaluated through crystallization experiments performed with citric acid in water. We acquired 2-D images using a new in situ imaging probe and treated them using an image analysis method composed of three steps. A segmentation step enabled us to identify each particle in an image (spatial support and focused/nonfocused particles). Thereafter, a restoration step was designed that enabled us to maximize the number of particles that could be processed in a reliable way. Such a goal was reached by means of the deconvolution of blurred particles. Finally, the last step (particle measurement) enabled us to obtain the PSD over time (up to moderate solid concentration). Experimental validation was performed through computer simulation and silica beads experiments. The proposed PSD imaging method was implemented in MATLAB and all the computations were performed on a personal computer with a 2-GHz CPU and $512 \mathrm{Mb}$ of RAM running windows XP. With such computer setup and after a few optimizations, the PSD imaging method required about 1s to complete an image. It is clear that such a processing time is too long to enable real-time PSD calculation. However, a conversion to the Clanguage and a multi-CPU optimization could drastically improve it. 
Journal of Electronic Imaging, 2010, 19(3), 0312071-7, Note: Special Section on Quality Control by Artificial Vision, doi: 10.1117/ 1.3462800

\section{Acknowledgements}

We greatly acknowledge the French research agency ANR for the support granted to the project "IPAPI" (Improving the Properties of Active Pharmaceutical Ingredients), reference 07-BLAN-0183.

\section{References}

[1] C. M. Keck and R. H. Müller, "Size analysis of submicron particles by laser diffractomery-90\% of the published measurements are false," Int. J . Pharm. 355, 150163 (2008).

[2] P. Mougin, "Sensitivity of particle sizing by ultrasonic attenuation spectroscopy to material properties," Powder Technol. 134, 243- 248 (2003).

[3] E. Hukkanen and R. Braatz, "Measurement of particle size distribution in suspension polymerization using is situ laser backscattering," Sens. Actuat. B Chem. 96, 451- 459 (2003).

[4] J. Worlitschek, T. Hocker, and M. Mazzotti, "Restoration of PSD from chord length distribution data using the method of projections onto convex sets," Part. Part. Syst. Charact. 22, 81- 98 (2005).

[5] J. Calderon de Anda, X. Z. Wang, and K. J. Roberts, "Multi-scale segmentation image analysis for the in-process monitoring of particle shape with batch crystallisers," Chem. Eng. Sci. 60, 1053-1065 (2005).

[6] P. A. Larsen, J . B. Rawlings, and N. J . Ferrier, "An alforithm for analyzing noisy, in situ images of high-aspect-ratio crystals to monitor particle size distribution," Chem. Eng. Sci. 61, 5236- 5248 (2006).

[7] Y. Zhou, R. Srinivasan, and S. Lakshminarayanan, "Critical evaluation of image processing approaches for real-time crystal size measurements," Comput. Chem. Eng. 33, 1022- 1035 (2009).

[8] D. D. Dunuwila, L. B. Carroll, and K. A. Berglund, "An investigation of the applicability of attenuated total reflection infrared spectroscopy for measurement of solubility and supersaturation of aqueous citric acid solutions," J . Cryst. Growth 137, 561- 568 (1994).

[9] F. Lewiner, J. P. Klein, F. Puel, and G. Févotte, "On-line ATR FTIR measurement of supersaturation during solution crystallization processes. Calibration and applications on three solute/ solvent systems," Chem. Eng. Sci. 56, 2069- 2084 (2001).

[10] S. Beucher, "The watershed transformation applied to image segmentation," Scanning Microsc. Int. 6, 299- 314 (1991).

[11] L. Vincent, "Morphological grayscale reconstruction in image analysis: applications and efficient algorithms," IEEE Trans. Image Process. 2, 176- 201 (1993).

[12] R. C. Gonzalez and R. E. Woods, Digital Image Processing, 2nd ed., Addison-Wesley Longman Publishing Co., Inc., Boston, MA (2002).

[13] P. T. Yap and P. Raveendran, "Image focus measurement based on Chebyshev moments," IEE Proc. Vision Image Signal Process. 151, 128- 136 (2004).

[14] J . Kautsky, J . Flusser, B. Zitová, and S. Simberová, "A new wavelet based measure of image focus," Pattern Recogn. Lett. 23, 1785- 1794 (2002).

[15] C.-Y. Wee and R. Paramesran, "Measure of image sharpness using eigenvalues," Inf. Sci. 177, 2533- 2552 (2007).

[16] M. Subbarao, T. Choi, and A. Nikzad, "Focusing techniques," J. Opt. Eng. 32, 28242836 (1993).

[17] B. Presles, "Suivi in situ de la distribution en taille et en forme de particules lors de leurs cristallisations en solution," MS degree, École Nationale Supérieure des Mines de SaintÉtienne (2008).

[18] D. Kundur and D. Hatzinakos, "Blind image deconvolution," IEEE Signal Process. Mag. 13, 43- 64 (1996). 
J ournal of Electronic Imaging, 2010, 19(3), 0312071-7, Note: Special Section on Quality Control by Artificial Vision, doi: 10.1117/ 1.3462800

[19] D. Kundur and D. Hatzinakos, "Blind image deconvolution revisited," IEEE Signal Process. Mag. 13, 61- 63 (1996).

[20] D. A. Fish, A. M. Brinicombe, E. R. Pike, and J. G. Walker, "Blind deconvolution by means of the Richardson-Lucy algorithm,"J . Opt. Soc. Am. A 12, 58- 65 (1995).

[21] B. Presles, J. Debayle, A. Rivoire, G. Févotte, and J .-C. Pinoli, "Monitoring the particle size distribution using image analysis during batch crystallization processes," in Proc. Int. Conf. on Quality Control by Artificial Vision, Wels, Austria (2009).

[22] P. Soille, Morphological Image Analysis: Principles and Applications, Springer-Verlag New York, Inc., Secaucus, NJ (2003).

[23] http:// www.beckmancoulter.com/ 
J ournal of Electronic Imaging, 2010, 19(3), 0312071-7, Note: Special Section on Quality Control by Artificial Vision, doi: 10.1117/ 1.3462800

\section{Figures}

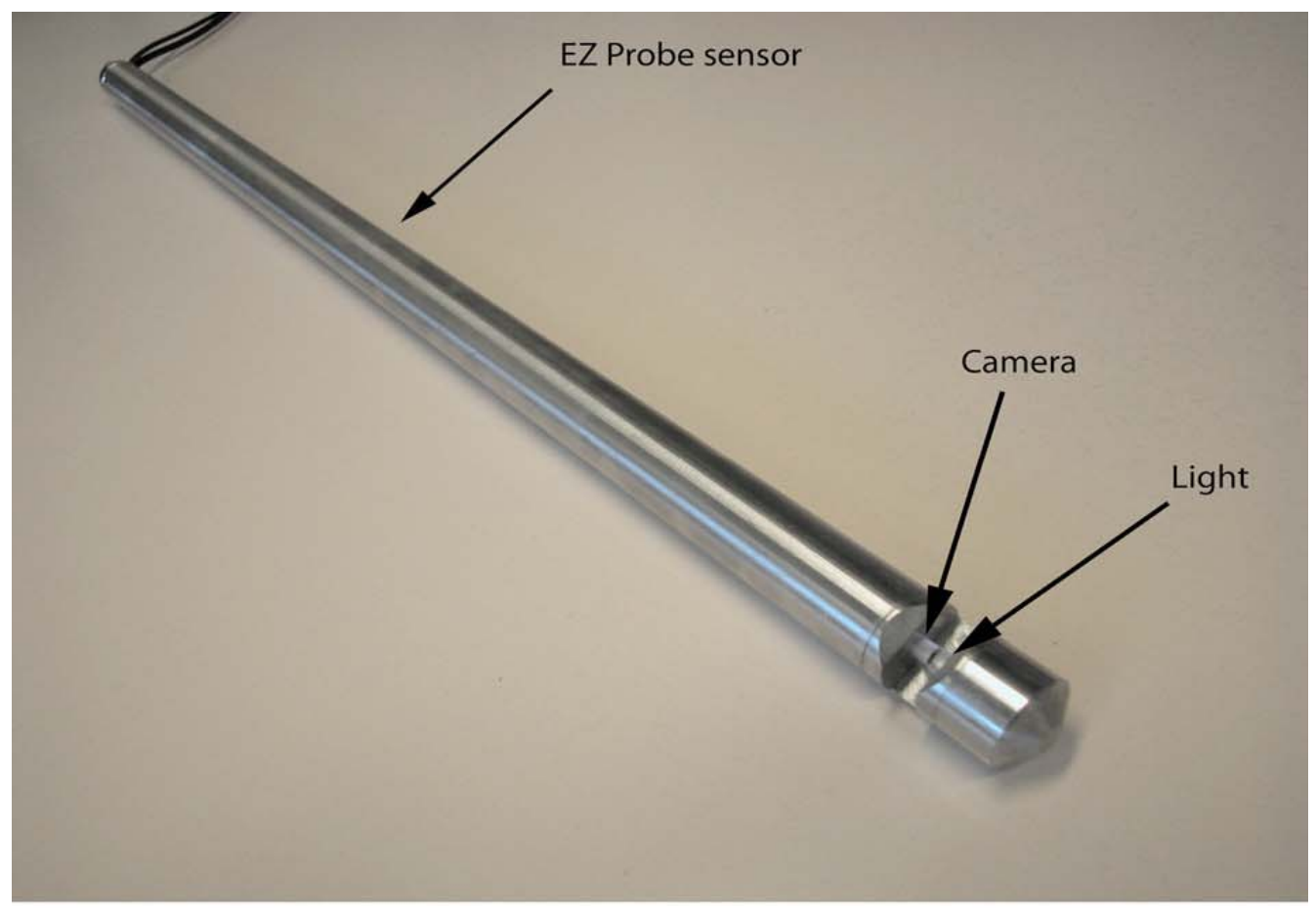

(a)

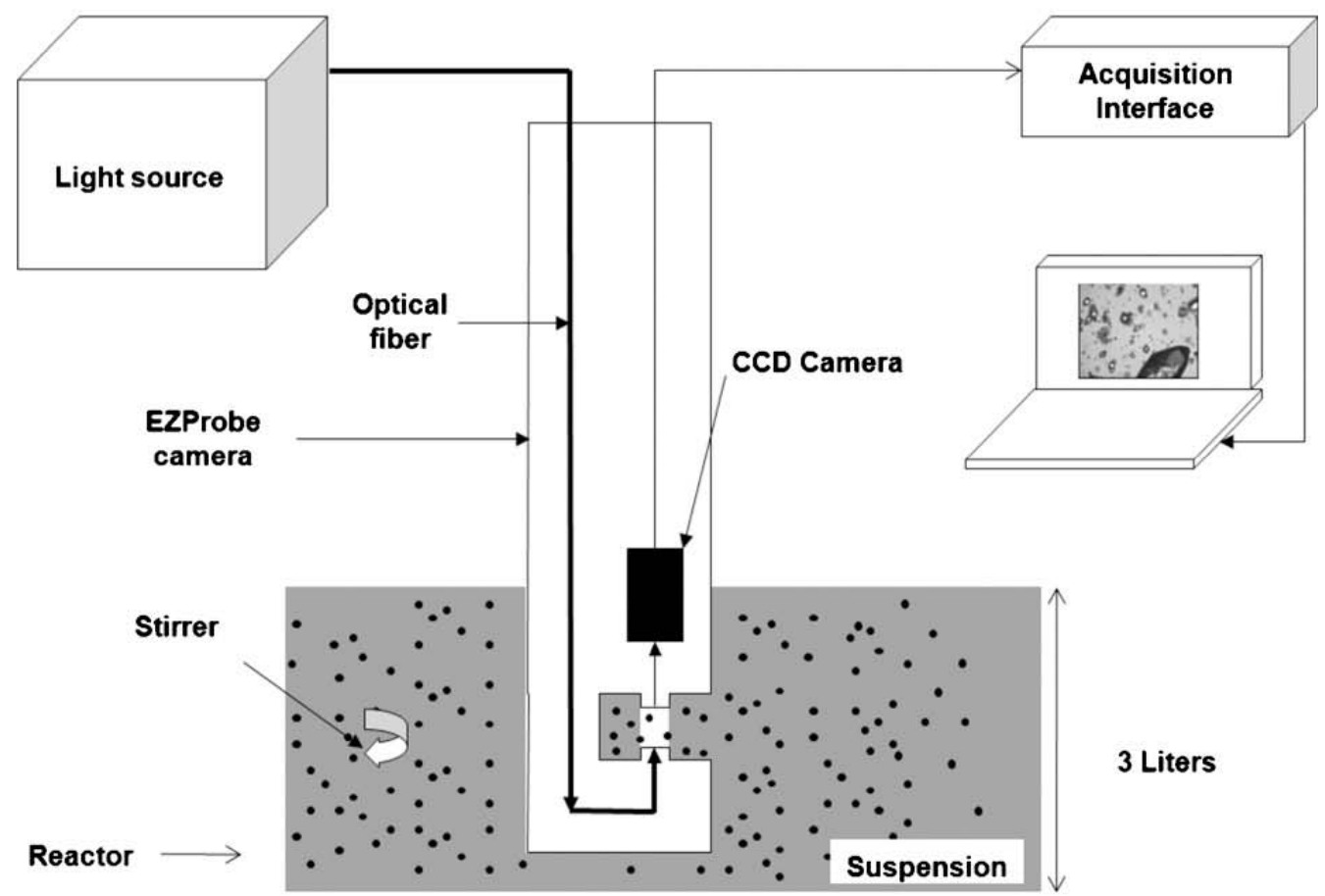

(b)

Figure 1: Experimental system: (a) EZProbe sensor and (b) schematic of the system. 
J ournal of Electronic Imaging, 2010, 19(3), 0312071-7, Note: Special Section on Quality Control by Artificial Vision, doi: 10.1117/ 1.3462800

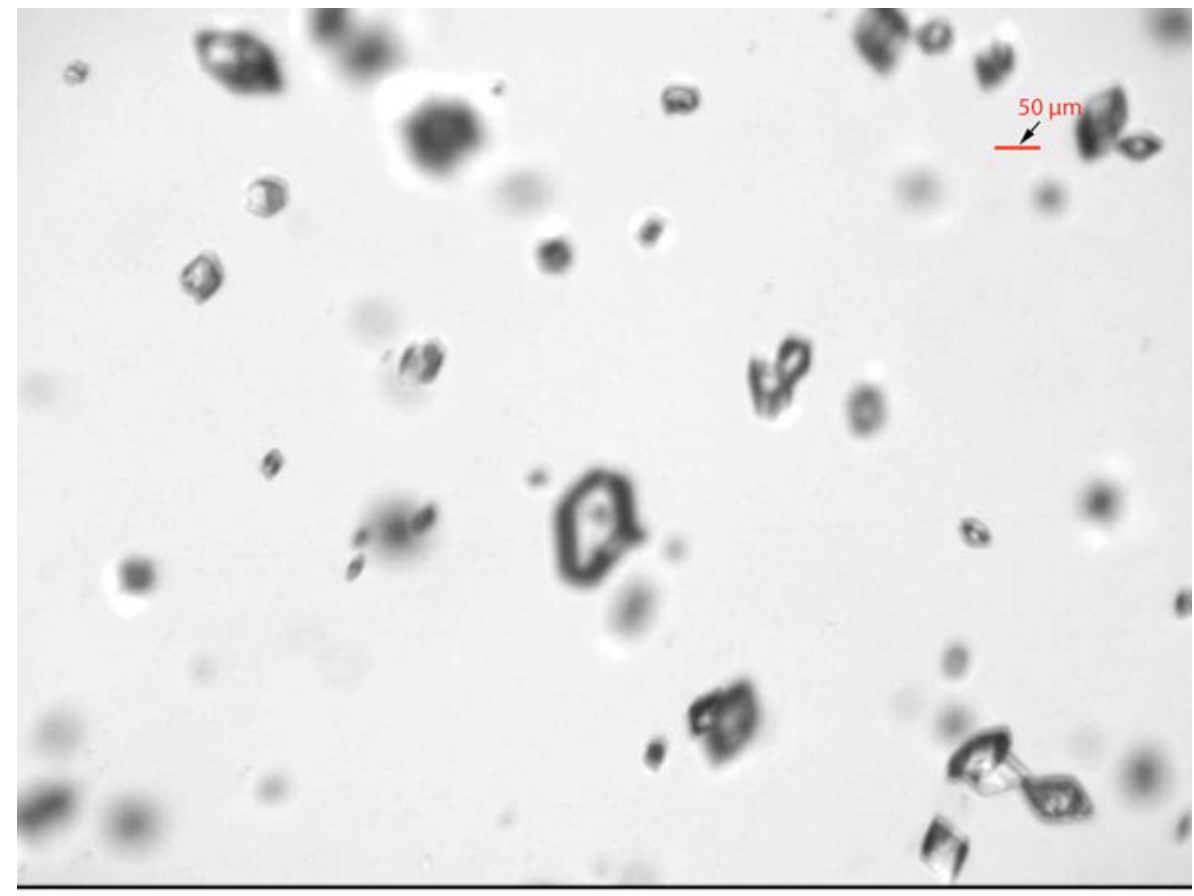

(a)

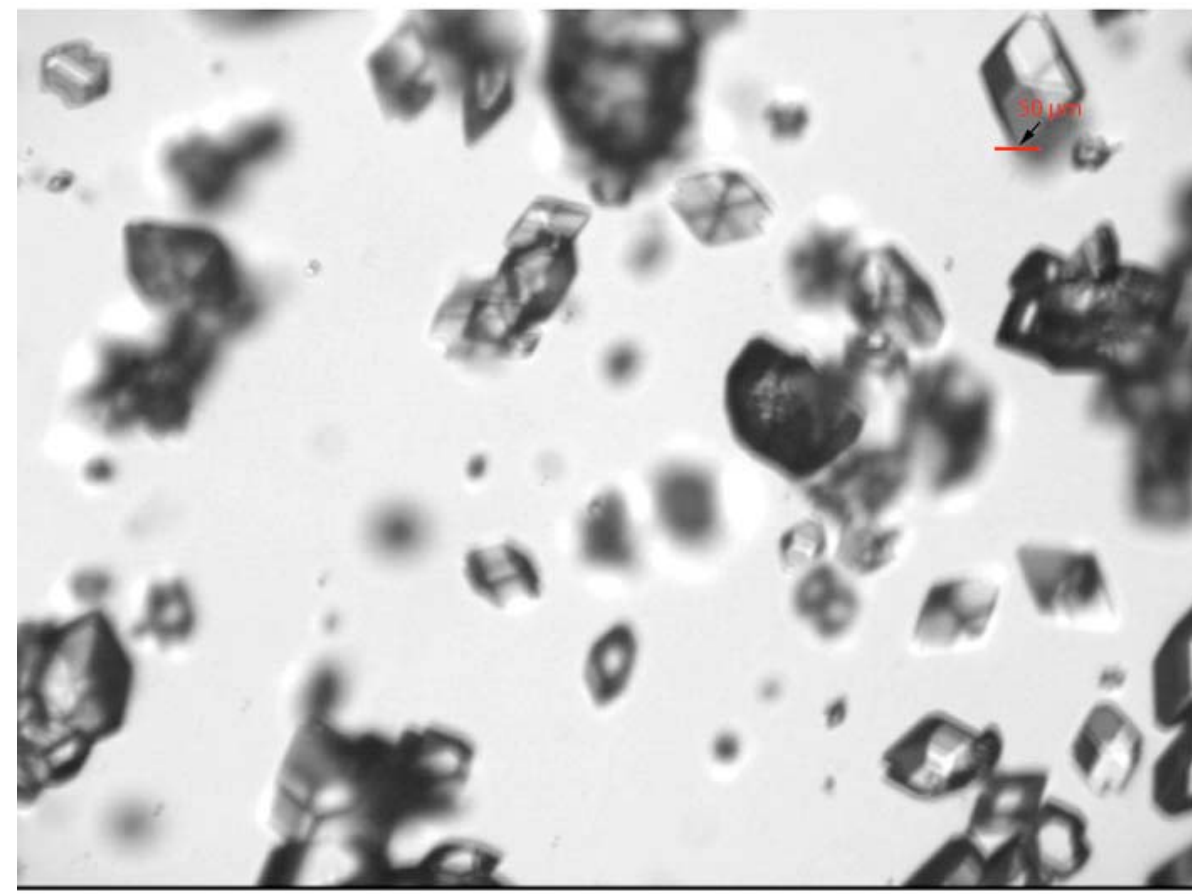

(b)

Figure 2: 2-D images of citric acid particles taken by the in situ imaging probe (a) $t=3$ min and (b) $t=11 \mathrm{~min}$. 
J ournal of Electronic Imaging, 2010, 19(3), 0312071-7, Note: Special Section on Quality Control by Artificial Vision, doi: 10.1117/ 1.3462800

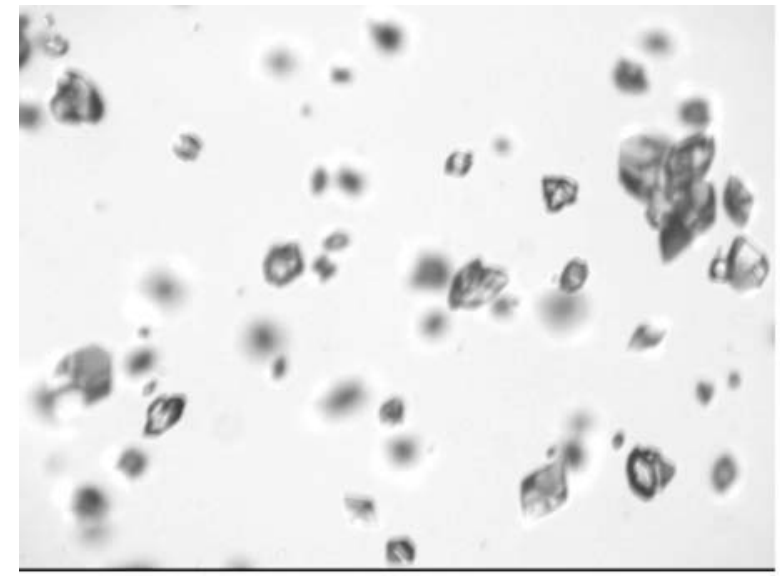

(a)

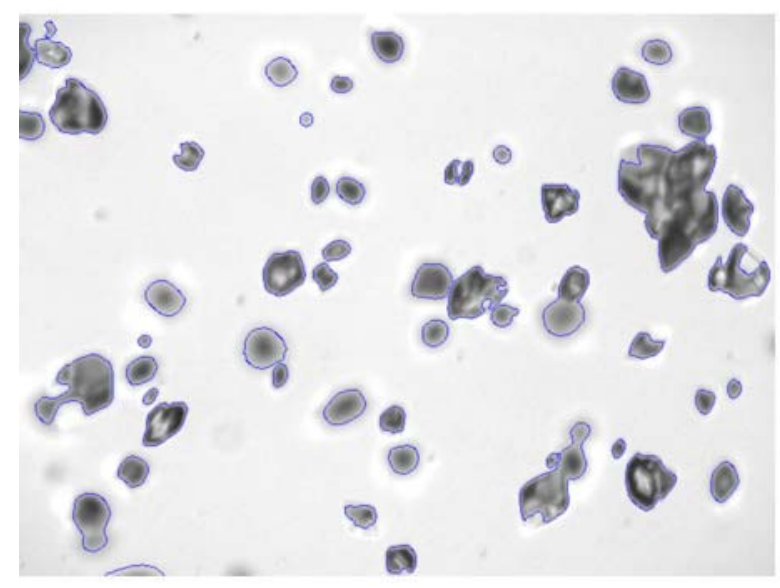

(c)

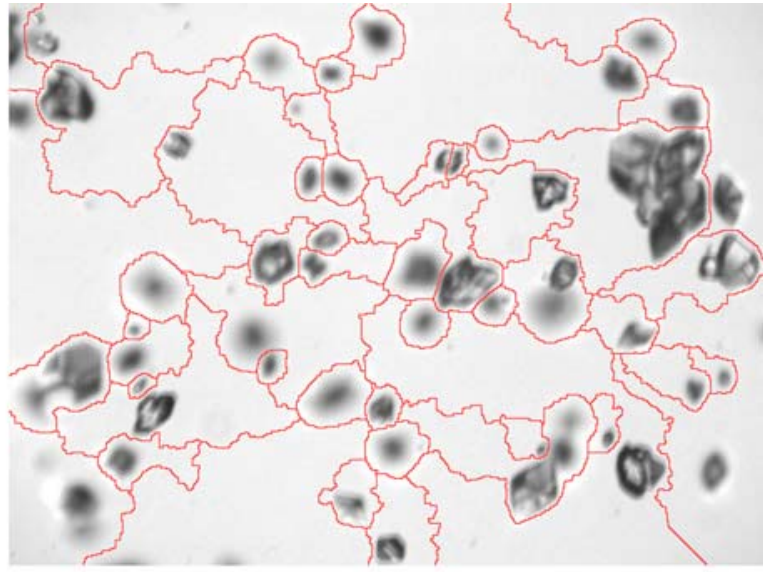

(b)

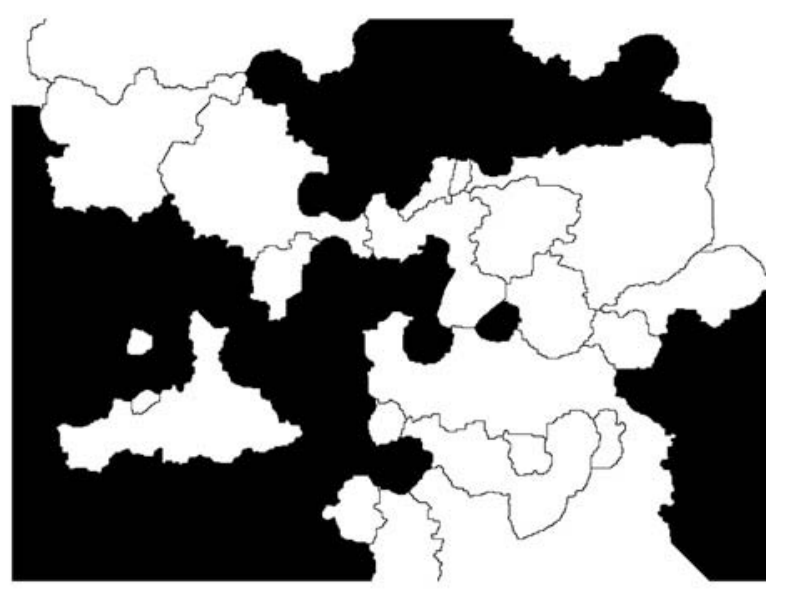

(d)

Figure 3: Segmentation of the particles image; The spatial support of each particle is detected and blurred and focused particles are discriminated: (a) an original image within a video sequence, (b) the constrained watershed algorithm applied to the original image, (c) spatial support of each particle (blue solid line), (d) blurred (black)/ focused (white) region/ particles. (Color online only). 
J ournal of Electronic Imaging, 2010, 19(3), 0312071-7, Note: Special Section on Quality Control by Artificial Vision, doi: 10.1117/ 1.3462800

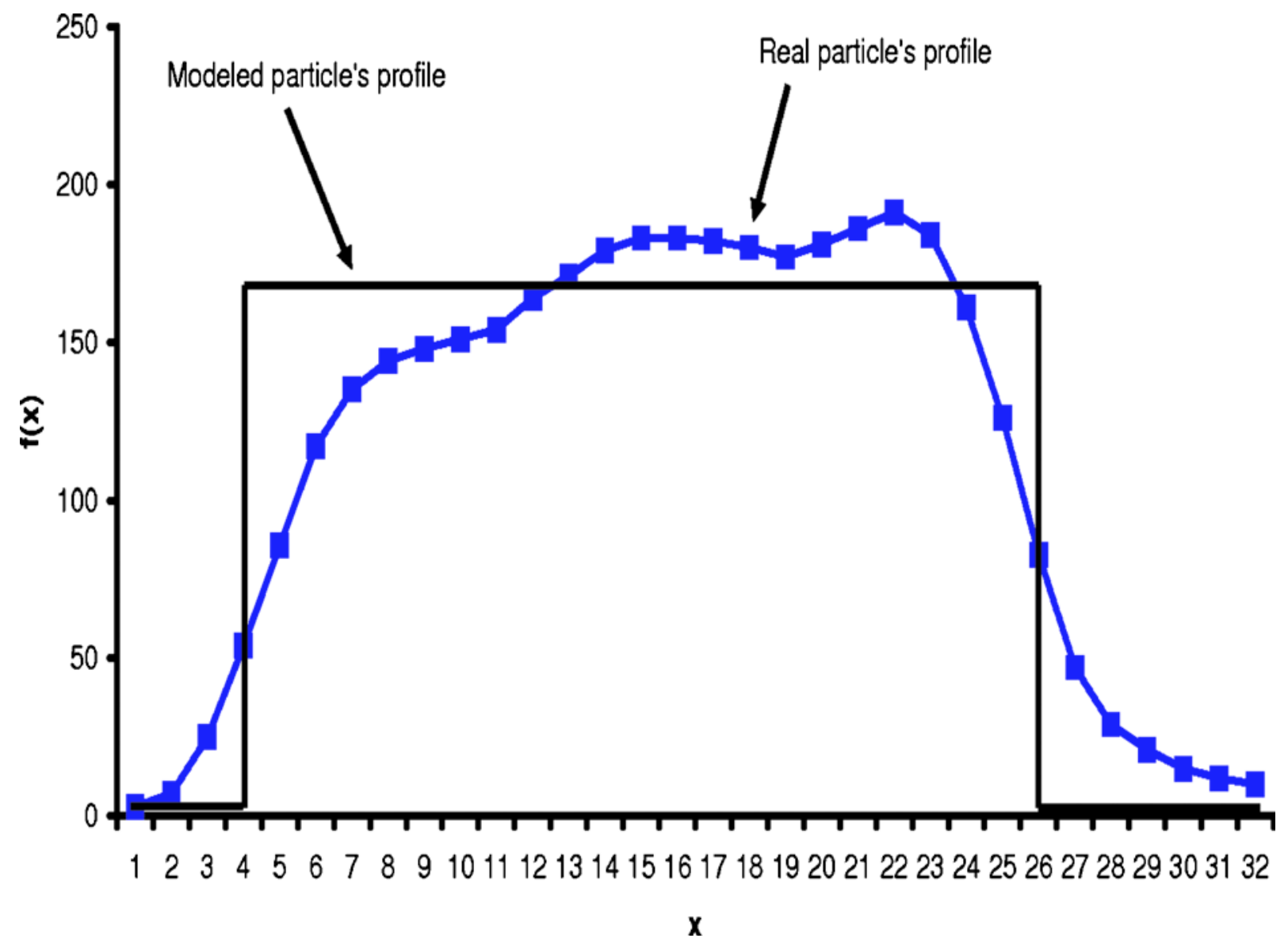

Figure 4: Real and modeled particle 1-D profile: blue squares indicate the profile of a real "perfect" (not blurred) particle image; in black, the corresponding squared modeled profile. (Color online only).

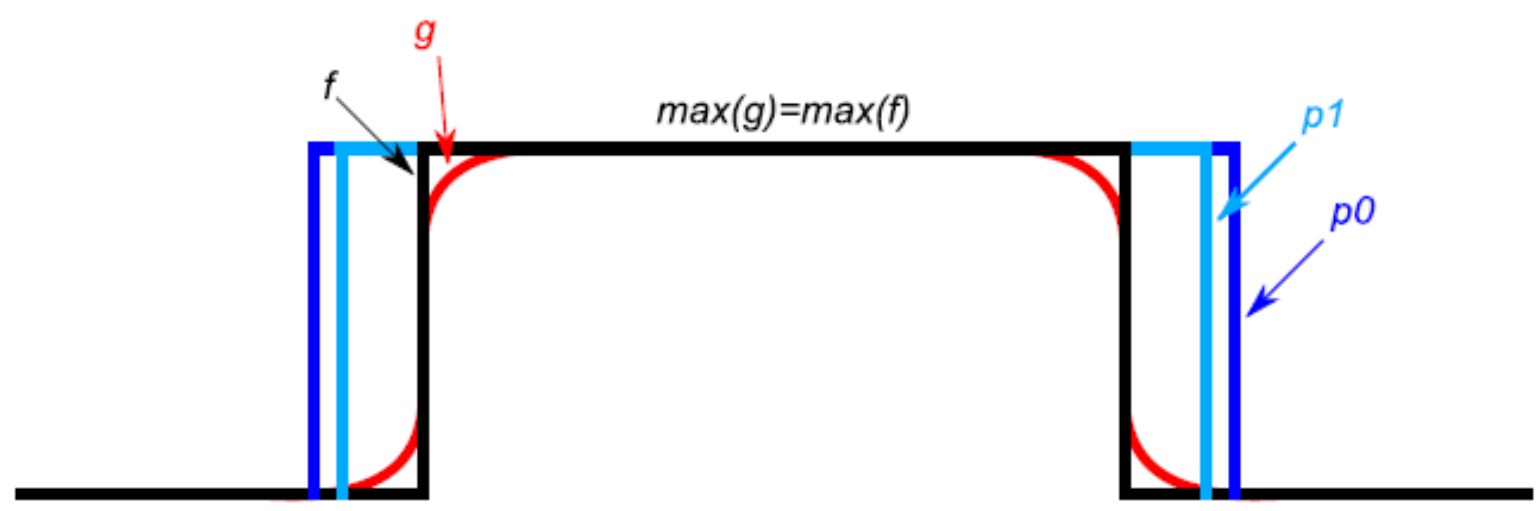

Figure 5: Iterative erosion of the spatial support of g: po, square function of "height" max (g) and spatial support g; p1, square function of "height" max (g) and spatial support g eroded once, etc. 
J ournal of Electronic Imaging, 2010, 19(3), 0312071-7, Note: Special Section on Quality Control by Artificial Vision, doi: 10.1117/ 1.3462800

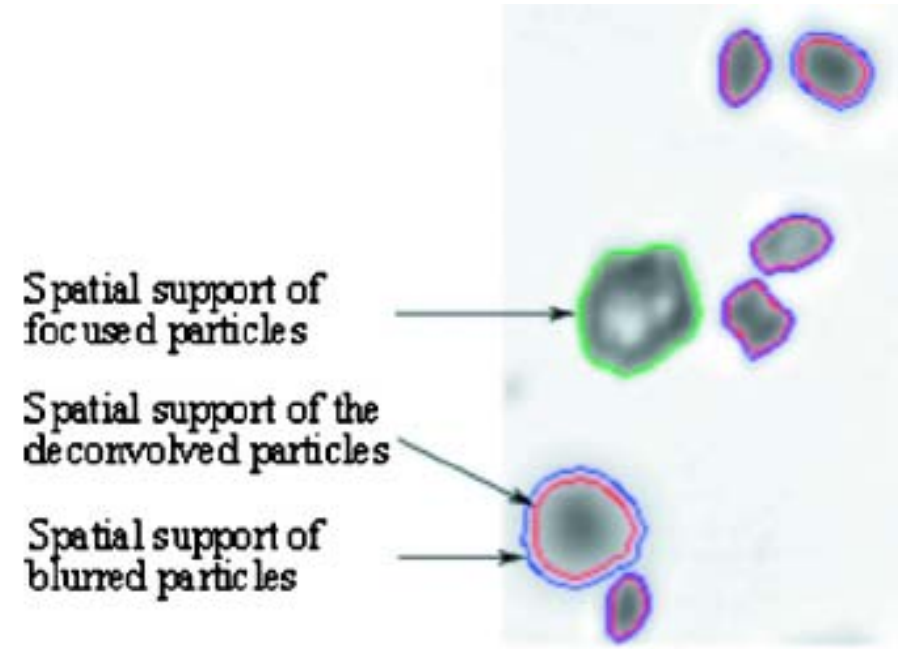

Figure 6: Restoration of the blurred particles (outside the focal plane) shown on a magnified image: in blue, spatial support of blurred particles; in green, spatial support of focused particles; and in red, spatial support of the deconvolved particles. (Color online only). 
J ournal of Electronic Imaging, 2010, 19(3), 0312071-7, Note: Special Section on Quality Control by Artificial Vision, doi: 10.1117/ 1.3462800

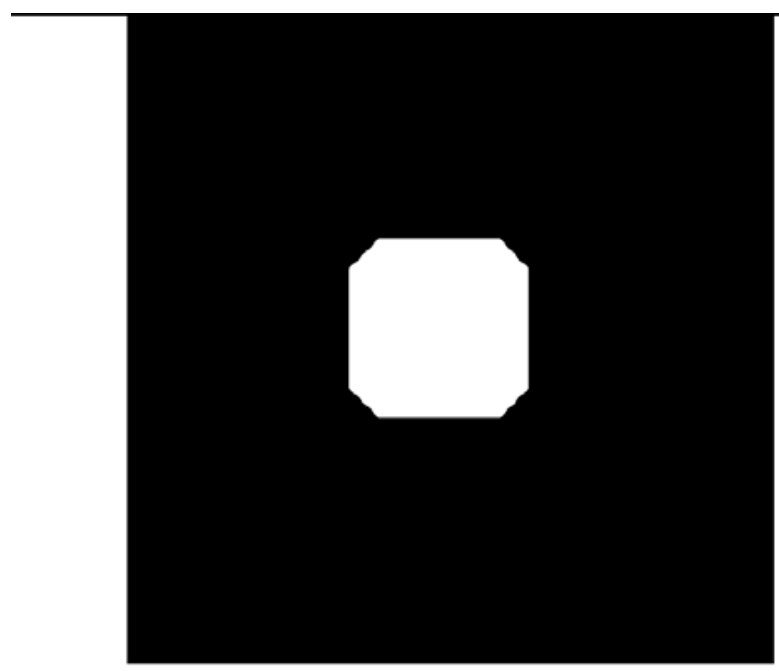

(a)

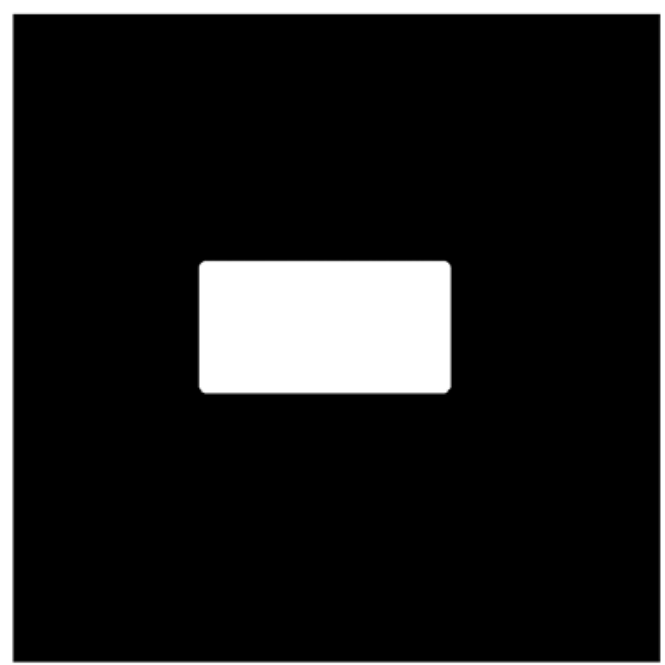

(b)

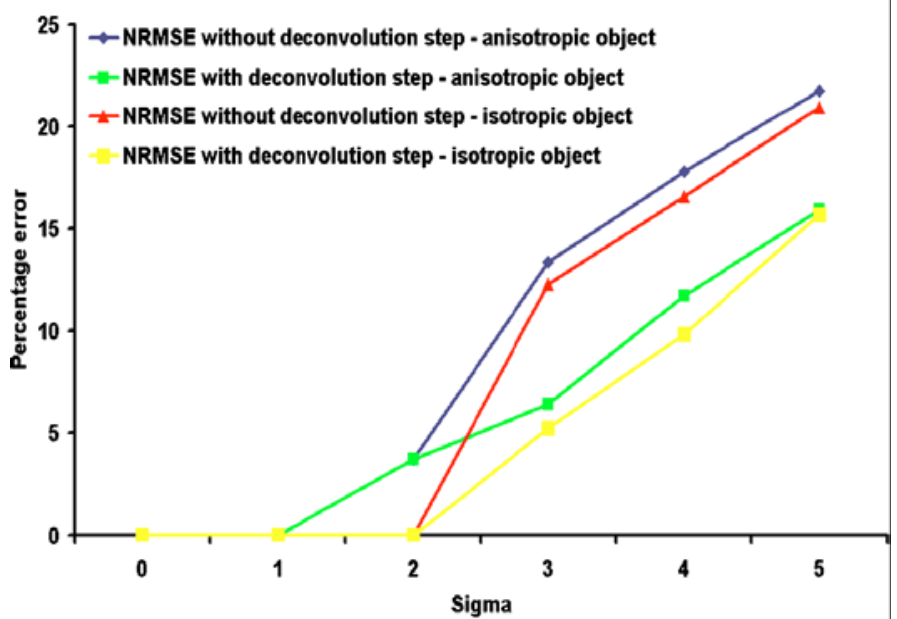

(c)

Figure 7: NRMSE as a function of sigma for (a) an isotropic and (b) an anisotropic objects and (c) calculated with and without the deconvolution step for the two objects. 
J ournal of Electronic Imaging, 2010, 19(3), 0312071-7, Note: Special Section on Quality Control by Artificial Vision, doi: 10.1117/ 1.3462800

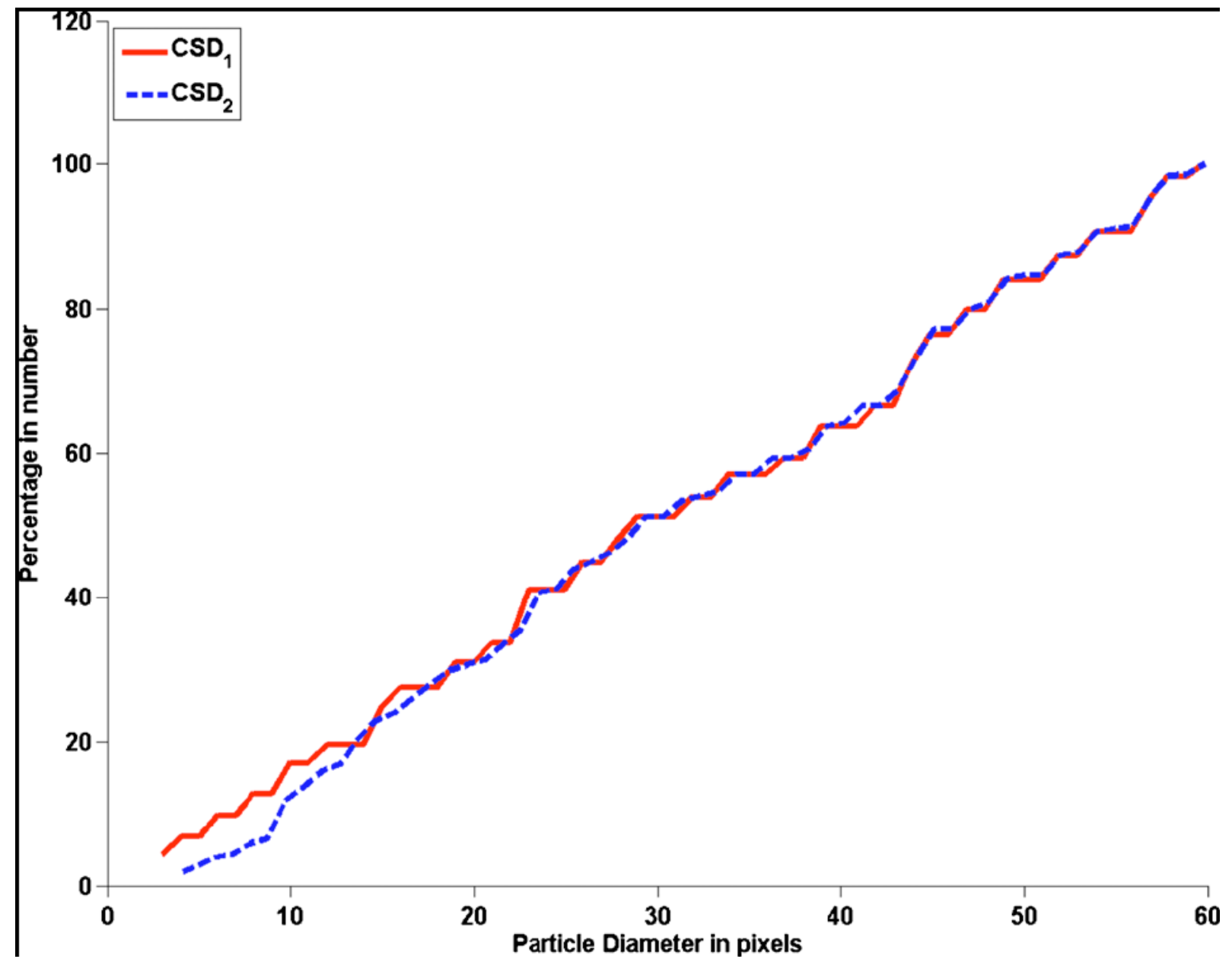

Figure 8: In red (solid line) the real CSD of the particles (CSD1); in blue (dashed line) the recovered CSD (CSD2). (Color online only). 
J ournal of Electronic Imaging, 2010, 19(3), 0312071-7, Note: Special Section on Quality Control by Artificial Vision, doi: 10.1117/ 1.3462800

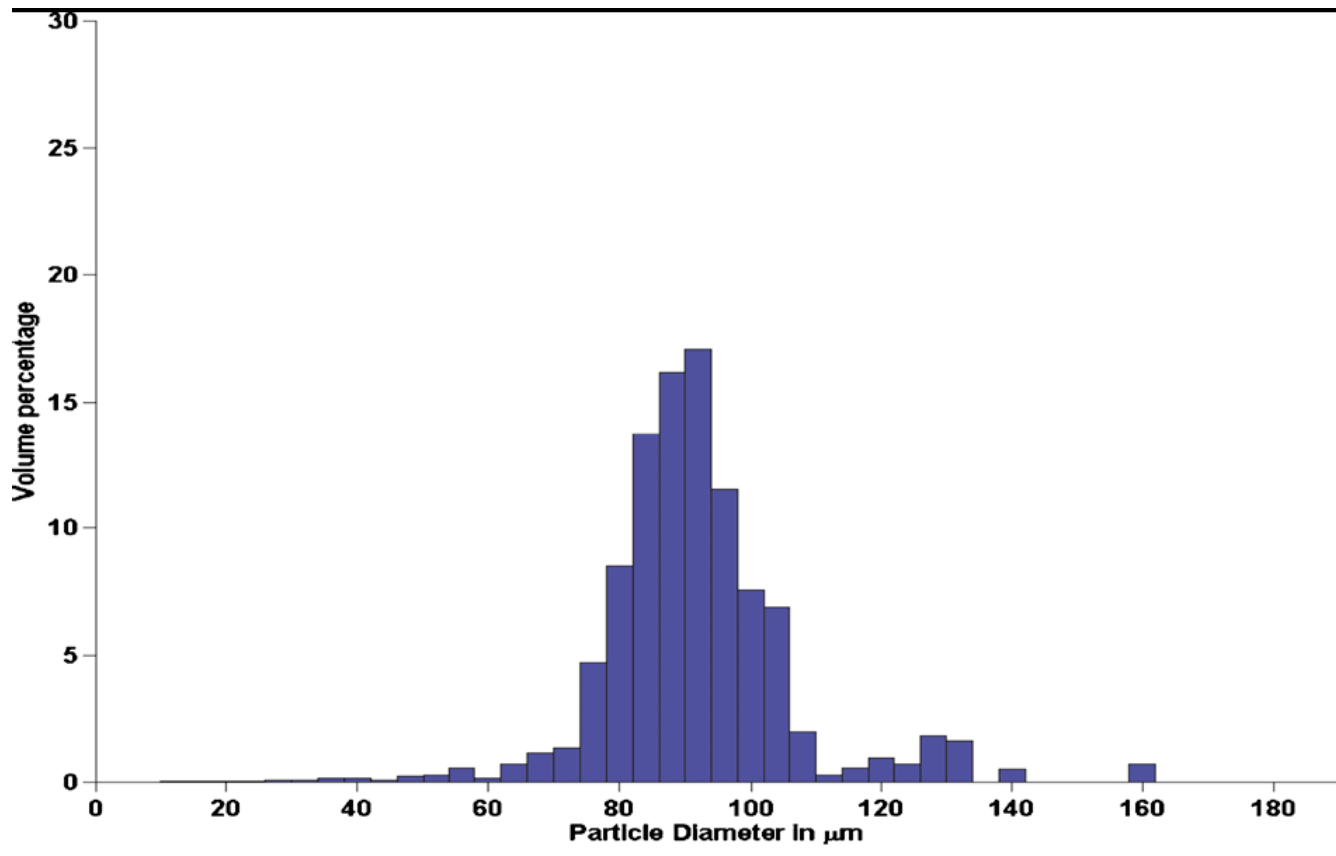

(a)

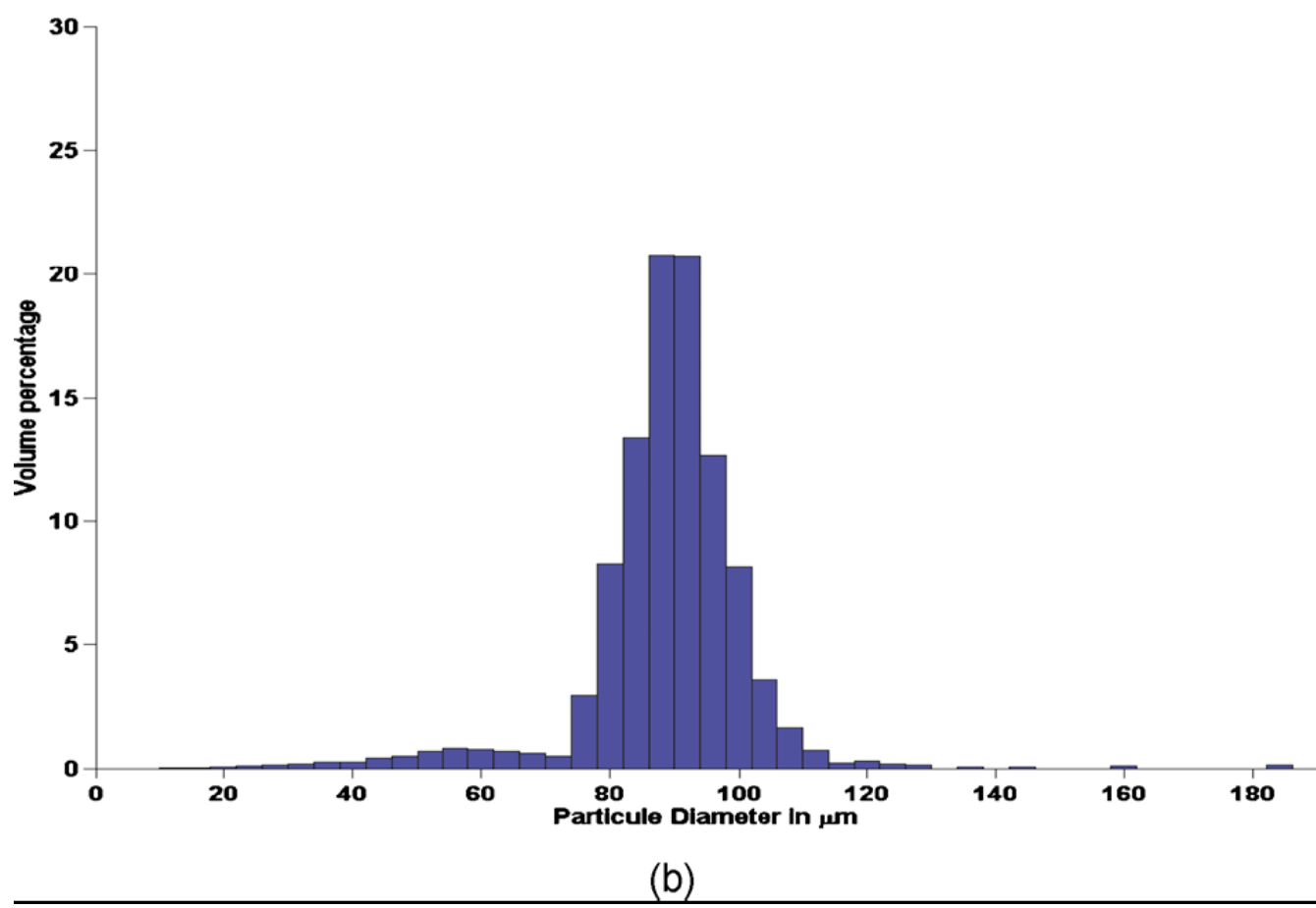

Figure 9: PSDs of the polydisperse silica beads obtained with (a) the PSD imaging method and (b) the Coulter counter method. PSDs are expressed in terms of volume percentage. 
J ournal of Electronic Imaging, 2010, 19(3), 0312071-7, Note: Special Section on Quality Control by Artificial Vision, doi: 10.1117/ 1.3462800

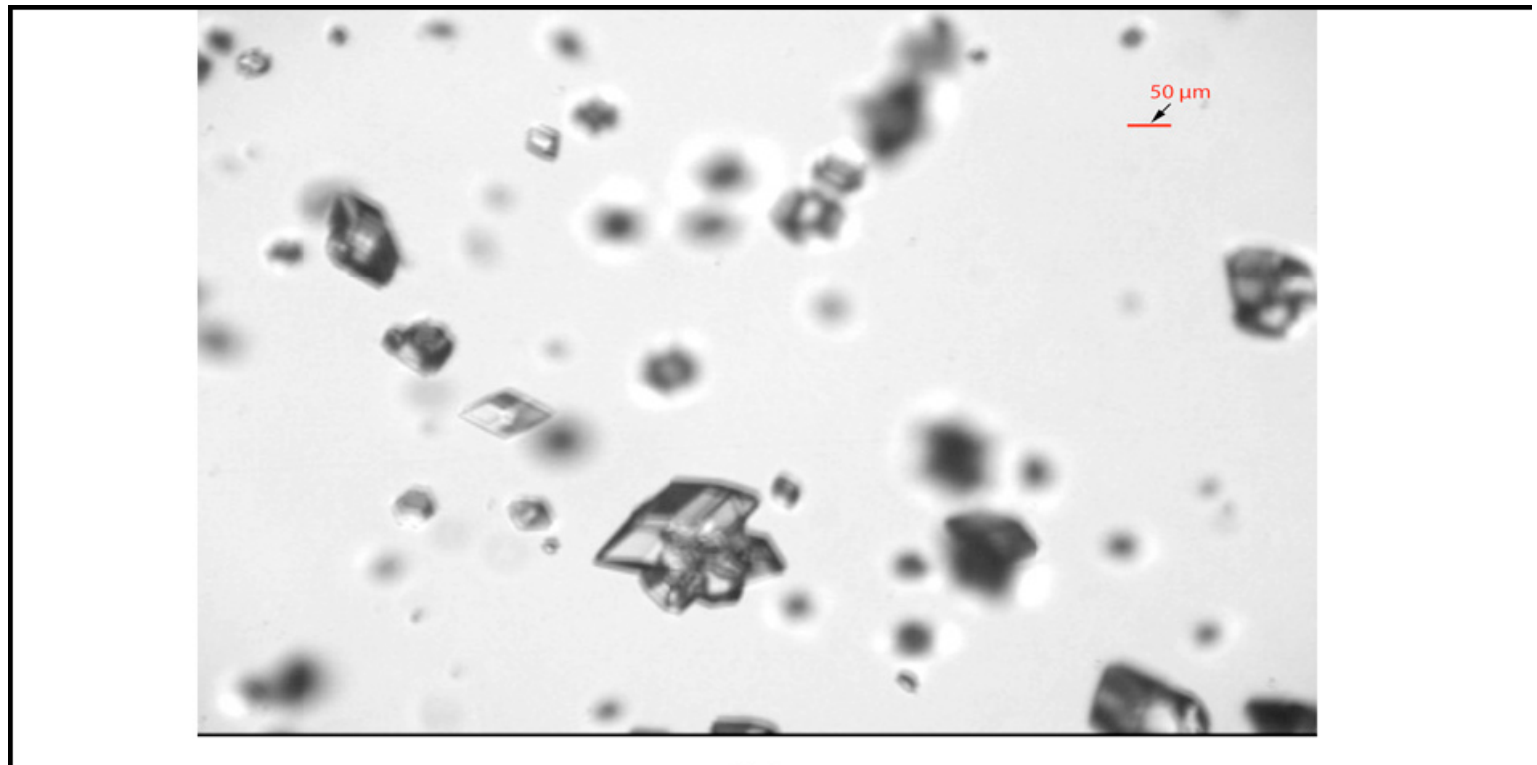

(a)

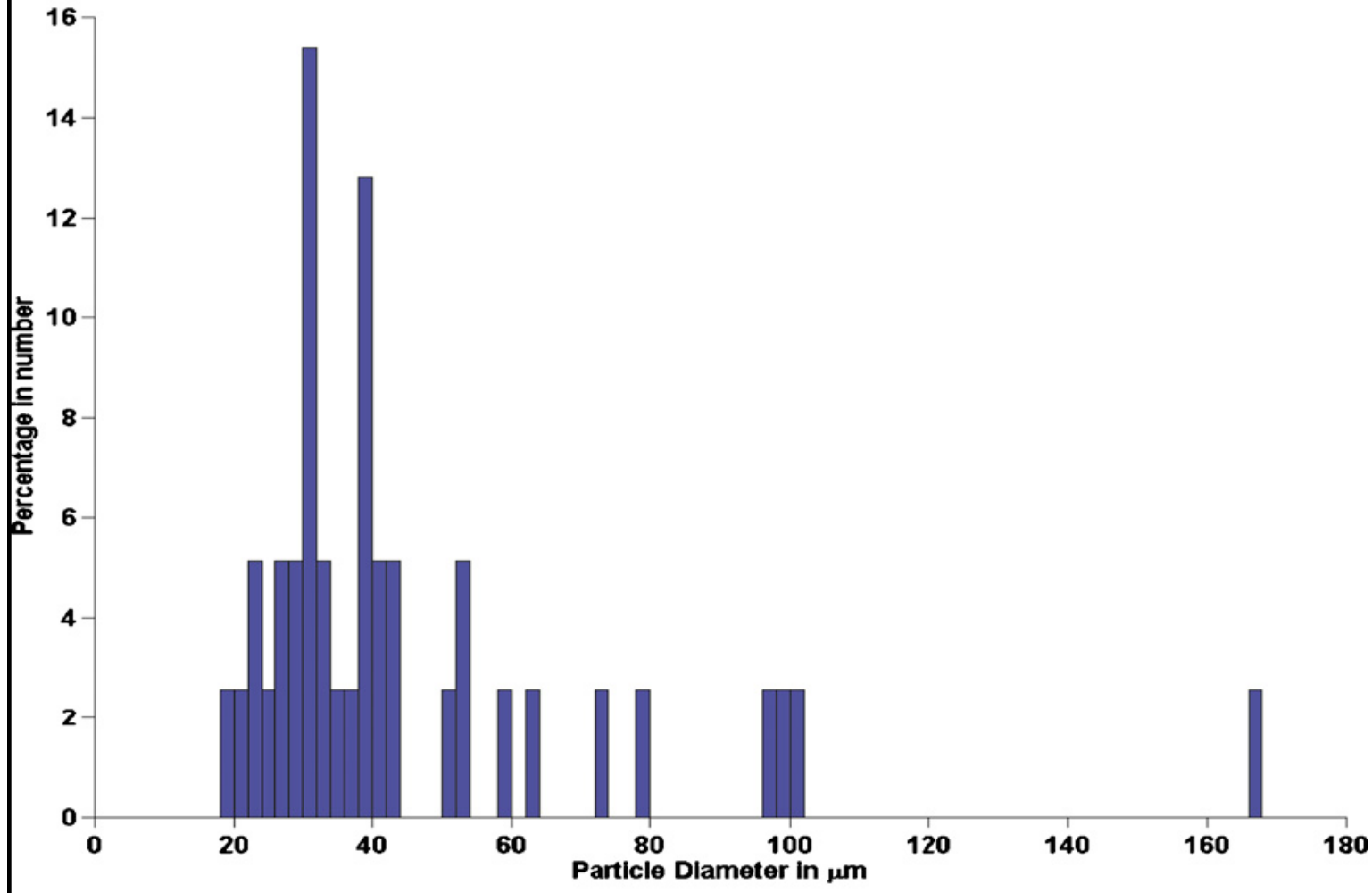

(b)

Figure 10: PSD of acid citric particles at $t=5 \min 23 \mathrm{~s}$ : (a) processed 2-D image and (b) PSD in number. The PSD is expressed in terms of percentage of number of particles. 
J ournal of Electronic Imaging, 2010, 19(3), 0312071-7, Note: Special Section on Quality Control by Artificial Vision, doi: 10.1117/ 1.3462800

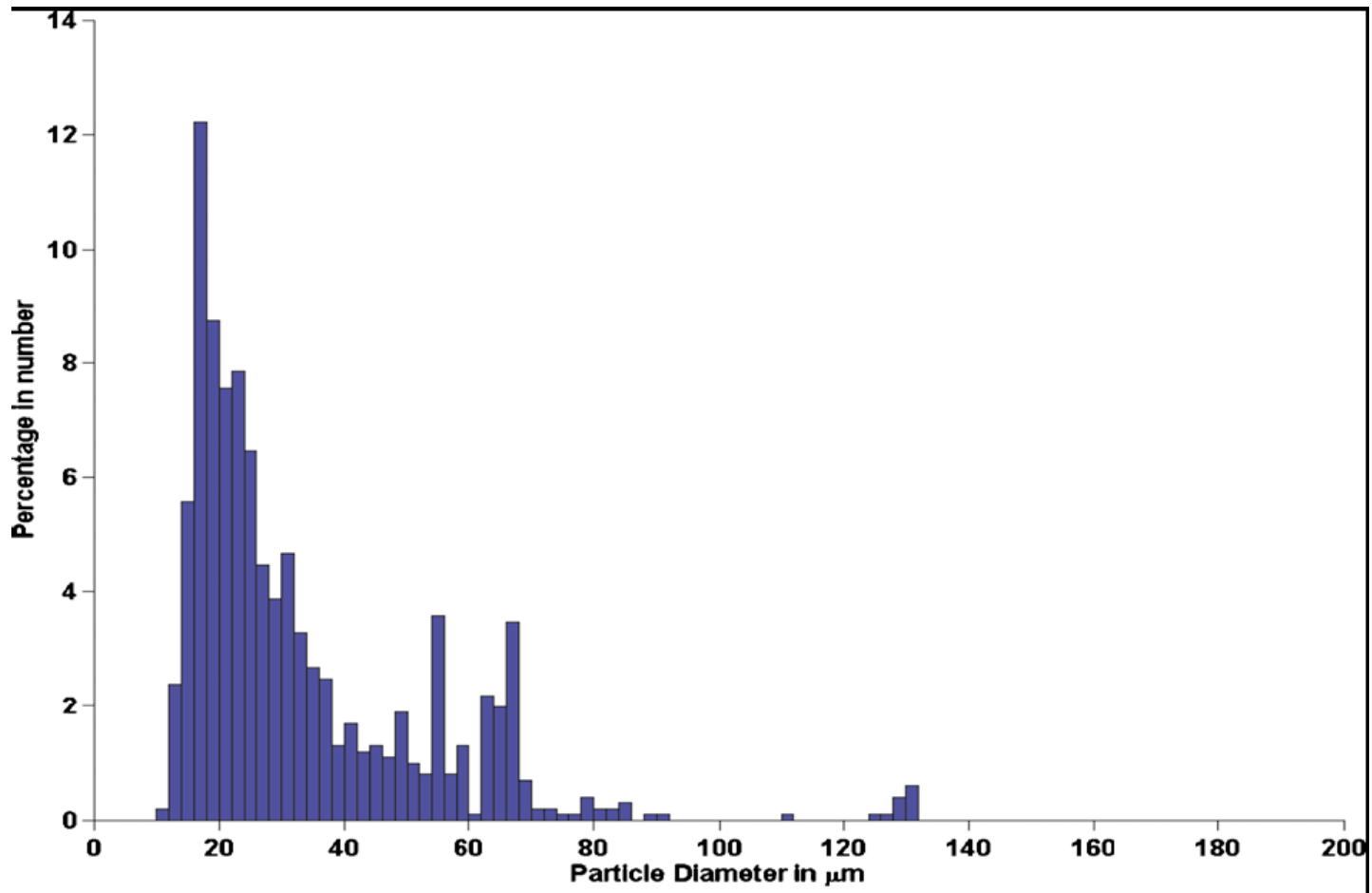

(a)

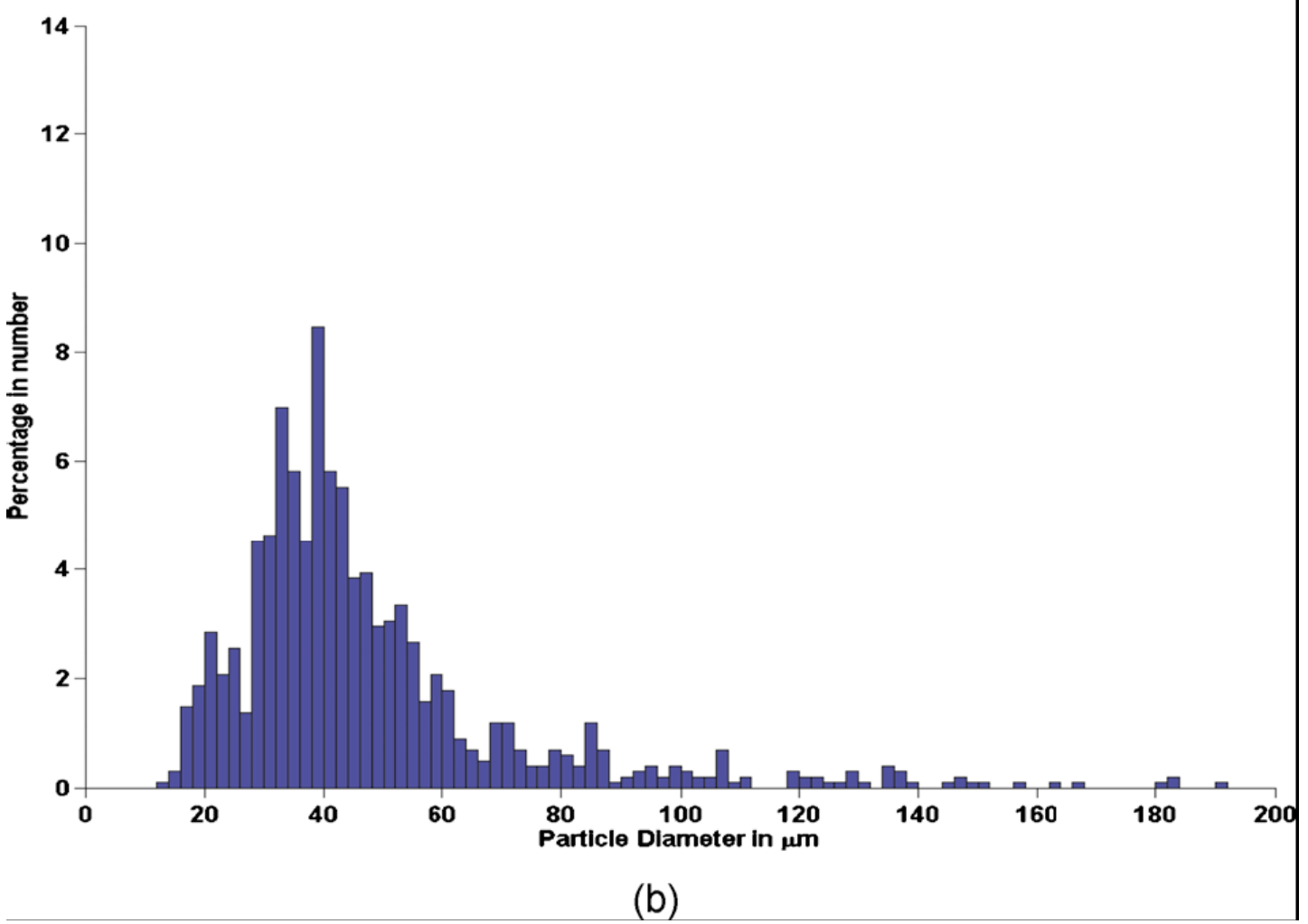

Figure 11: Average PSD after about (a) 2 min and (b) 5 min of crystallization. The PSDs are expressed in terms of percentage in number of particles. 